\title{
Perceptions and Behaviors of Patients with Inflammatory Bowel Disease during the COVID-19 Crisis
}

\author{
Yoo Jin Lee ${ }^{1}$, Kyeong Ok Kim², Min Cheol Kim², Kwang Bum Cho'1, Kyung Sik Park², and Byeong Ik Jang², on \\ behalf of the Crohn's and Colitis Association in Daegu-Gyeongbuk (CCAiD) \\ ${ }^{1}$ Department of Internal Medicine, Keimyung University School of Medicine, and ${ }^{2}$ Department of Internal Medicine, Yeungnam \\ University College of Medicine, Daegu, Korea
}

\author{
Article Info \\ Received February 10, 2021 \\ Revised May 1, 2021 \\ Accepted May 18, 2021 \\ Published online July 12, 2021
}

Corresponding Author

Kyeong Ok Kim

ORCID https://orcid.org/0000-0001-5799-7436

E-mail kokim@yu.ac.kr
Background/Aims: This study aimed to investigate the perceptions and behaviors of patients with inflammatory bowel disease (IBD) during the early coronavirus disease 2019 (COVID-19) pandemic in the major epidemic area in Korea.

Methods: Between April and May 2020, a cross-sectional survey was performed at two tertiary hospitals in Daegu, South Korea, on patients' experiences, coping strategies and perceptions.

Results: Most of the 544 patients participating in the survey strictly adhered to personal protection guidance against COVID-19. In the early COVID-19 crisis, many patients canceled or postponed hospital visits $(57.5 \%)$ and rescheduled biologics administrations (26.4\%). Although $13.6 \%$ utilized telemedicine, the frequency of individuals leaving their homes remained unchanged. Although $50.4 \%$ were concerned about their susceptibility to COVID-19, $72.2 \%$ adhered to their treatment for IBD. In patients taking biologics or Janus kinase inhibitors, $86.8 \%$ disagreed that they should discontinue their medication as a preventative measure against COVID-19, but $21.9 \%$ actually discontinued their medications. Of those with discordance between the perception of IBD drug adherence and active behaviors, $5.4 \%$ of all and $39.4 \%$ of biologics or Janus kinase inhibitors withheld drugs specifically due to fear of COVID-19. Only $7 \%$ of all patients discussed drug safety with their physicians. The level of concern for COVID-19 depended on the type of drug used.

Conclusions: Patients with IBD showed concerns about the increased risk of COVID-19 due to IBD or their medications, and a considerable number of patients withheld their medications without consulting medical staff. Enhanced active communications with patients with IBD and appropriate health-related education should be provided. (Gut Liver 2022;16:81-91)

Key Words: Inflammatory bowel diseases; COVID-19; Perception; Medication adherence

\section{INTRODUCTION}

The rapid spread of coronavirus disease 2019 (COVID-19) has significantly impacted all fields of medicine. In South Korea, the COVID-19 outbreak emerged from Daegu on February 18, 2020, centering on a certain religious group. Subsequently, the number of confirmed COVID-19 patients rapidly increased in Daegu in the southeastern region of South Korea. ${ }^{1}$ Because of the rapid increase in confirmed cases, with the highest incidence and mortality in South Korea, sudden, repeated emergency department closures occurred in Daegu. ${ }^{2}$ When an infectious disease outbreak occurs, treatment of chronic diseases can be affected. Although it is vital to reduce the risk of COVID-19 exposure in patients with chronic immune diseases such as inflammatory bowel disease (IBD), it is also important to manage the underlying disease appropriately to prevent worsening when access to emergency medical care may be difficult. Evaluation of patients' thoughts and behaviors regarding the situation in the epidemic area is needed to understand the appropriate management strategy for patients with IBD. Therefore, we aimed to investigate the percep- 
tions and behaviors of patients with IBD during the early COVID-19 crisis in the region of South Korea initially affected the most by the epidemic.

\section{MATERIALS AND METHODS}

This study is a telephone-based, cross-sectional survey performed at two tertiary hospitals in Daegu, South Korea. All patients with IBD who had visited the hospitals within 3 months from the start of this study were eligible to participate. These patients were previously registered in a prospective IBD registry (registered at ClinicalTrial.gov, NCT02193464) and had agreed to provide demographic and contact information for future research purposes. Patients who did not respond to the phone call, declined to participate, or were unable to respond to the survey system were excluded.

We defined the early COVID-19 crisis as occurring from February 21, 2020 to March 31, 2020, based on the daily confirmed cases in Daegu. The survey was conducted between April 24, 2020 and May 22, 2020, after approval by the institutional review board. Two investigators independently conducted the telephone calls. After obtaining permission from the participants, their behaviors in daily life and coping strategies were assessed through telephone surveys. To obtain clearer answers on perceptions, the subsequent questionnaires were collected anonymously through a web-based system (PADAS software, PADAS Co. Ltd., Seoul, Korea) via links sent through a text message after the telephone call. The contents of the questionnaire are presented in Supplementary Material 1. Patient demographics, disease locations, and behaviors using the Montreal classification, ${ }^{3}$ laboratory data, and drug therapies were extracted from the prospectively collected database.

The primary outcome was to investigate the perceptions and behaviors of patients with IBD during the early COVID-19 crisis. The secondary outcome was to evaluate the differences in patients' perceptions according to the different IBD drugs in the epidemic region. We also evaluated the disparity between patient perceptions and coping strategies regarding drug adherence.

\section{Survey questionnaire and proceedings}

The questionnaires mainly consisted of the following three categories: (1) experiences and practices toward COVID-19 in daily life (seven questions), (2) coping strategies for hospital resource utilization and IBD drugs (12 questions), and (3) perspectives of COVID-19 and IBD (seven questions). To increase the response rate, most questions were short and close-ended and could be answered by "yes" or "no." The questions regarding respondents' perceptions were scored on a 5-point scale, with the following choices: strongly disagree, disagree, neutral, agree, and strongly agree. If the web-based questionnaire was not returned within 1 week, reminders for the completion of the questionnaire were sent through a text message. We closed the survey link 2 weeks after it was sent out.

\section{Statistics}

Categorical variables were presented as frequencies with percentages and analyzed using the Fisher exact test; continuous variables were expressed as means and standard deviations and analyzed using the independent $t$-test; twosided p-values of 0.05 were considered statistically significant. Statistical analysis was performed using the Statistical Package for the Social Sciences (SPSS) version 23.0 (IBM Corp., Armonk, NY, USA).

\section{Ethical considerations}

The study was conducted in accordance with the Declaration of Helsinki and was approved by the Institutional Review Board of Yeungnam University Hospital (IRB number: 2020-04-034) and registered at ClinicalTrial.gov (NCT04387279). All participants provided written informed consent.

\section{RESULTS}

\section{Demographics and patient characteristics}

Among the 1,002 patients who were registered in our prospective IBD registry, 879 adults who visited within 3 months prior to the survey were eligible to participate in this study. After excluding patients who did not respond to the phone call $(n=198)$, declined to participate $(n=112)$, or were unable to respond to the survey system $(\mathrm{n}=25)$, a total of 544 patients completed the survey (response rate $61.9 \%$, 544/879). The patients consisted of 206 men (37.9\%) with a mean age of 40 years. The baseline and clinical characteristics of the patients are shown in Table 1 . The number of patients with Crohn's disease and ulcerative colitis was 255 (46.9\%) and 289 (53.1\%) patients, respectively. Of the 289 patients with ulcerative colitis, left-sided colitis was the most common in 105 (36.3\%), followed by pancolitis in $102(35.3 \%)$, and proctitis in $82(28.4 \%)$. Of the 255 Crohn's disease patients, A2 ( $\mathrm{n}=192,75.3 \%), \mathrm{L} 3$ ( $\mathrm{n}=137$, $53.7 \%)$, and B1 ( $\mathrm{n}=149,58.4 \%)$ were observed according to the Montreal classification. ${ }^{3}$ The most commonly used medication was 5-aminosalicylate (5-ASA), which accounted for $76.3 \%$ of participants, followed by immuno- 
Table 1. Demographics and Clinical Characteristics of Patients with Inflammatory Bowel Disease

\begin{tabular}{|c|c|}
\hline Characteristics & Value $(n=544)$ \\
\hline Age, mean $\pm S D$, yr & $40.34 \pm 15.31$ \\
\hline Male sex & 206 (37.9) \\
\hline \multicolumn{2}{|l|}{ Occupation } \\
\hline Office worker & $219(40.3)$ \\
\hline Self-employment & 68 (12.5) \\
\hline Students & $79(14.5)$ \\
\hline Housewife & $74(13.6)$ \\
\hline Unemployment or retired & 104 (19.1) \\
\hline Duration of disease, median (range), yr & $5.17(0.36-36.00)$ \\
\hline \multicolumn{2}{|l|}{ Disease type } \\
\hline$C D$ & 255 (46.9) \\
\hline UC & $289(53.1)$ \\
\hline \multicolumn{2}{|l|}{ UC extension } \\
\hline E1 & $82(28.4)$ \\
\hline E2 & 105 (36.3) \\
\hline E3 & $102(35.3)$ \\
\hline \multicolumn{2}{|l|}{$C D$, age at diagnosis } \\
\hline A1 & $31(12.2)$ \\
\hline A2 & 192 (75.3) \\
\hline A3 & 32 (12.5) \\
\hline \multicolumn{2}{|l|}{$C D$, location } \\
\hline L1 & 77 (30.3) \\
\hline L2 & $41(16.1)$ \\
\hline L3 & $137(53.7)$ \\
\hline \multicolumn{2}{|l|}{$\mathrm{CD}$, behavior } \\
\hline B1 & $149(58.4)$ \\
\hline B2 & $67(26.3)$ \\
\hline B3 & 39 (15.3) \\
\hline $\mathrm{p}$ & 75 (29.4) \\
\hline \multicolumn{2}{|l|}{ UC, disease activity* } \\
\hline Remission & $244(84.4)$ \\
\hline Mild & 40 (13.8) \\
\hline Moderate & $4(1.4)$ \\
\hline Severe & $1(0.3)$ \\
\hline \multicolumn{2}{|l|}{ CD, disease activity ${ }^{\dagger}$} \\
\hline Very well & 213 (83.5) \\
\hline Mild activity & $26(10.2)$ \\
\hline Moderate & $16(6.3)$ \\
\hline Severe & 0 \\
\hline \multicolumn{2}{|l|}{ Medication at the time of survey } \\
\hline 5-ASA & 415 (76.3) \\
\hline 5-ASA suppository & $137(25.2)$ \\
\hline Immunomodulators (azathioprine, 6-MP, MTX) & 206 (37.9) \\
\hline Corticosteroid & $26(4.8)$ \\
\hline Biologics or JAK inhibitors & $174(32.0)$ \\
\hline Infliximab & 103 (18.9) \\
\hline Adalimumab & $37(6.8)$ \\
\hline Ustekinumab & $16(2.9)$ \\
\hline Vedolizumab & $14(2.6)$ \\
\hline Tofacitinib & $4(0.7)$ \\
\hline 5-ASA alone & $232(42.6)$ \\
\hline Biologics+immunomodulator & 68 (12.5) \\
\hline Immunomodulator alone & $138(25.4)$ \\
\hline Biologics alone or JAK inhibitors & 106 (19.5) \\
\hline
\end{tabular}

Table 1. Continued

\begin{tabular}{lc}
\hline \multicolumn{1}{c}{ Characteristics } & Value $(\mathrm{n}=544)$ \\
\hline Laboratory finding within 3 months from & \\
survey date, mean $\pm S D, m g / d L$ & \\
CRP & $0.45 \pm 1.17$ \\
ESR & $20.35 \pm 17.88$ \\
Albumin & $4.45 \pm 0.64$ \\
\hline
\end{tabular}

Data are presented as number (\%) unless otherwise indicated.

CD, Crohn's disease; UC, ulcerative colitis; 5-ASA, 5-aminosalicylic acid; 6-MP, 6-mercaptopurine; MTX, methotrexate; JAK, Janus kinase; CRP, C-reactive protein; ESR, erythrocyte sedimentation rate. Montreal classification of UC; disease extension: E1 (proctitis), E2 (left-sided), E3 (extensive). Montreal classification of CD; age at diagnosis: A1 (below 16 years), A2 (between 17 and 40 years), A3 labove 40 years); disease location: L1 (terminal ileum), L2 (colon), L3 (ileocolon), L4 (upper gastrointestinal tract); disease behavior: B1 (nonstricturing nonpenetrating), B2 (structuring), B3 (penetrating), p (perianal disease modifier).

*Assessed by partial mayo score among UC patients ( $n=289)$ : remission 0-1, mild 2-4, moderate 5-6, severe 7-9; ${ }^{\dagger}$ Assessed by Crohn's Disease Activity Index among CD patients $(n=255)$ : remission $<150$, mild 150-220, moderate 220-450, severe $>450$.

modulators (37.9\%). Approximately one-third of respondents were treated with biologics or Janus kinase (JAK) inhibitors ( $\mathrm{n}=174,32.0 \%)$.

\section{Experiences and coping behavior during the COVID-19 crisis in daily life}

Table 2 shows patients' experiences and coping behaviors toward COVID-19 in their daily lives during the COVID-19 epidemic. Most patients strictly wore masks (98.1\%) and maintained social distancing (95.8\%). Approximately $15 \%$ of patients experienced voluntary business closures in forms of voluntary departures or reduced working hours. Approximately 7\% of patients received a polymerase chain reaction test for COVID-19, and no IBD patients were infected with severe acute respiratory syndrome coronavirus 2 (SARS-CoV-2) in our study. Moreover, $2.8 \%$ of patients underwent self-quarantine according to South Korean government's COVID-19 response system. A few patients (8.5\%) had experienced symptoms consistent with SARSCoV-2 infection. Less than half of them (20/46, 43.5\%) discussed their symptoms of suspected COVID-19 with their physicians.

\section{Changes in hospital resource utilization}

During the COVID-19 crisis, $57.5 \%$ of patients had canceled or postponed hospital visits due to the following: fears of COVID-19 (59.7\%), personal reasons (37.1\%), and other reasons (3.2\%). Only $13.6 \%$ of patients answered that they had used telemedicine, which was temporarily allowed in South Korea during the COVID-19 pandemic period. Telemedicine satisfied $91.9 \%$ of patients who had experienced it; 
Table 2. Experiences and Coping Behaviors toward COVID-19 in Daily Lives ( $\mathrm{n}=544)$

\begin{tabular}{|c|c|}
\hline Questionnaires & No. $(\%)(n=544)$ \\
\hline \multicolumn{2}{|l|}{ I strictly wore masks when going out } \\
\hline Strong disagree & 0 \\
\hline Disagree & 0 \\
\hline Neutral & $10(1.8)$ \\
\hline Agree & $23(4.2)$ \\
\hline Strongly agree & 511 (93.9) \\
\hline \multicolumn{2}{|l|}{ I strictly maintained social distancing } \\
\hline Strong disagree & 0 \\
\hline Disagree & 0 \\
\hline Neutral & $23(4.2)$ \\
\hline Agree & 105 (19.3) \\
\hline Strongly agree & 416 (76.5) \\
\hline Experience of voluntary business closures (voluntary departures or reduced working hours) & $81(14.9)$ \\
\hline The duration of voluntary business closures, median (range), day* & $30(2-29)$ \\
\hline Experience of self-quarantine according to the government's COVID-19 response system & $15(2.8)$ \\
\hline Experience of symptoms consistent with COVID-19 & $46(8.5)$ \\
\hline \multicolumn{2}{|l|}{ Self-reported symptoms consistent with COVID-19 (multiple choice) } \\
\hline Coryza & $9(1.7)$ \\
\hline Cough & $14(2.6)$ \\
\hline Throat pain & $11(2.0)$ \\
\hline Fever & $13(2.4)$ \\
\hline Dyspnea & $3(0.6)$ \\
\hline Patients who had discussed their symptoms of suspected COVID-19 with physicians ${ }^{\dagger}$ & $20 / 46(43.5)$ \\
\hline Patients who had received the PCR test for COVID-19 & $40(7.4)$ \\
\hline
\end{tabular}

COVID-19, coronavirus disease 2019; PCR, polymerase chain reaction.

*Asked in 81 patients who experienced voluntary business closures; ${ }^{\dagger}$ Asked in 46 patients who experienced symptoms consistent with COVID-19.

Table 3. Patients' Coping Aspects for Hospital Resource Utilization ( $n=544)$

\begin{tabular}{|c|c|}
\hline Questionnaires & No./No. $(\%)$ \\
\hline Patients who had canceled or postponed hospital visits & $313 / 544(57.5)$ \\
\hline \multicolumn{2}{|l|}{ The reason for cancelation or delay in hospital visits* } \\
\hline Fears of COVID-19 & $187 / 313(59.7)$ \\
\hline Personal reasons & $116 / 313(37.1)$ \\
\hline Other reason (transportation, policy from workplace etc.) & $10 / 313(3.2)$ \\
\hline Patients who had used telemedicine & $74 / 544(13.6)$ \\
\hline \multicolumn{2}{|l|}{ Satisfaction for telemedicine service } \\
\hline Bad & 0 \\
\hline Soso & $1 / 74(1.4)$ \\
\hline I don't know & $5 / 74(6.8)$ \\
\hline Satisfied & $38 / 74(51.4)$ \\
\hline Very satisfied & $30 / 74(40.5)$ \\
\hline \multicolumn{2}{|l|}{ Purchasing prescription drugs following a telemedicine service ${ }^{+}$} \\
\hline Near the hospital & $53 / 74(71.6)$ \\
\hline Near the residence & $21 / 74(28.4)$ \\
\hline Self-reported IBD disease worsening during COVID-19 crisis & $91 / 544(16.7)$ \\
\hline Patients who had visited hospitals when self-reported IBD disease worsening ${ }^{\ddagger}$ & $36 / 91(39.6)$ \\
\hline
\end{tabular}

COVID-19, coronavirus disease 2019; IBD, inflammatory bowel disease.

*Asked in 313 patients who had canceled or postponed hospital visits; ${ }^{\dagger}$ Asked in 74 patients who had used telemedicine; ${ }^{\ddagger}$ Asked in 91 patients who answered that their IBD had worsened during the COVID-19 crisis.

however, the majority (71.6\%) answered that they had subsequently visited a pharmacy near the hospital to purchase prescription drugs. Although about $17 \%$ of patients an- swered that their IBD had worsened during the COVID-19 crisis, $39.6 \%$ of them visited the hospital (Table 3). 


\begin{tabular}{l} 
Q1: IBD patients are more susceptible to COVID-19 \\
Q2: COVID-19 can cause more severe lung injury \\
in IBD patients \\
\cline { 2 - 6 } Q
\end{tabular}

Fig. 1. Patients' perceptions on the risk of COVID-19 and IBD.

IBD, inflammatory bowel disease; COVID-19, coronavirus disease 2019; SARS-CoV-2, severe acute respiratory syndrome coronavirus 2.

\section{Perceptions of IBD and therapy during the COVID-19 crisis}

The degree of agreement for questions related to perceptions about IBD and therapy during the COVID-19 crisis (Q1-7) is presented in Fig. 1. Half of the IBD patients (50.4\%) perceived that they were more susceptible to COVID-19. However, the responses for specific concerns (Q2) to lung injury and intestinal damage (Q3) were relatively evenly distributed across the five levels of agreement. In contrast, more than half of the patients with IBD (65.6\%) disagreed that they should avoid hospital visits, even if the disease worsened (Q4). Most patients (83.8\%) disagreed that they should discontinue their IBD drugs to prevent COVID-19 (Q5). Similarly, 66.3\% of patients were against discontinuing IBD drugs, even if they were infected with SARS-CoV-2 (Q6). To the question about whether the pneumococcal vaccination will help prevent progression to severe pneumonia when infected with SARS-CoV-2, the numbers of patients who agreed, disagreed, and were neutral were comparable (Q7) (Fig. 1).

\section{Pattern of IBD drug usage}

A total of $27.8 \%$ of patients answered that they had discontinued IBD medications; however, only $7.9 \%$ of those discontinued their medication due to fears of COVID-19. Meanwhile, among those taking biologics or JAK inhibitors, about $26.4 \%$ reported that they had postponed or withheld the administration of biologics or JAK inhibitors.
The most common reason for rescheduling the administration of biologics or JAK inhibitors was fears of COVID-19 (47.8\%), followed by personal reasons (32.6\%), forgetting to take medication (4.3\%), and others (4.3\%); $13.0 \%$ did not respond. Only 7\% of patients had discussed about the safety of their IBD drug with their physician (Table 4).

\section{Disparities between patients' perceptions and behaviors regarding adherence to IBD treatment}

The results of the disparities between patient perceptions and coping aspects for drug adherence are presented in Fig. 2. The proportions of patients who disagreed that they should stop IBD drugs to prevent COVID-19 were similar between all patients $(456 / 544,83.8 \%)$ and patients taking biologics or JAK inhibitors (151/174, 86.8\%). The degrees of discordance between the perception of IBD drug administration and actual behavior were also similar between all patients $(112 / 456,24.6 \%)$ and patients taking biologics or JAK inhibitors (33/151, 21.9\%). However, when we considered the cause of nonadherence, $5.4 \%$ of all patients and $39.4 \%$ of those taking biologics/JAK inhibitors answered that the reason for their nonadherence to the drug was fear of COVID-19.

\section{Degree of agreement regarding perceptions}

A total of $66.0 \%$ of patients using biologic monotherapy or JAK inhibitors and $64.7 \%$ of those using biologics combined with immunomodulators agreed that "IBD patients 
Table 4. Respondents' Coping Aspects for IBD Drugs ( $n=544$ )

\begin{tabular}{lc}
\hline \multicolumn{1}{c}{ Questionnaires } & No./No. (\%) \\
\hline Patients who had discontinued IBD medications & $151 / 544(27.8)$ \\
The reason for discontinuation of IBD medications* & $12 / 151(7.9)$ \\
Fears of COVID-19 & $13 / 151(8.6)$ \\
Personal reasons & $98 / 151(64.9)$ \\
Forgotten & $2 / 151(1.3)$ \\
Others & $26 / 151(17.2)$ \\
Do not answer & $46 / 174(26.4)$ \\
Patients who had postponed or withheld biologics or JAK-inhibitors ${ }^{\dagger}$ & \\
The reason for rescheduling the administration of biologics or JAK-inhibitor ${ }^{\ddagger}$ & $22 / 46(47.8)$ \\
$\quad$ Fears of COVID-19 & $15 / 46(32.6)$ \\
Personal reasons & $2 / 46(4.3)$ \\
Forgotten & $2 / 46(4.3)$ \\
Others & $6 / 46(13.0)$ \\
Do not answer & $38 / 544(7.0)$ \\
Patients who had discussed about the safety of their IBD medications with physician &
\end{tabular}

IBD, inflammatory bowel disease; COVID-19, coronavirus disease 2019; JAK, Janus kinase.

${ }^{*}$ Asked in 151 patients who had discontinued IBD medications; ${ }^{\dagger}$ Asked in 174 patients taking biologics or JAK-inhibitors; ${ }^{\ddagger}$ Asked in 46 patients who had rescheduled the administration of biologics or JAK-inhibitors.

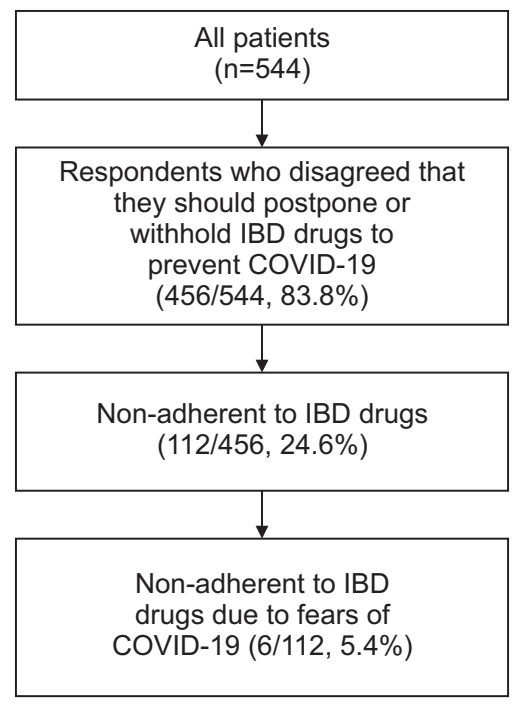

are more susceptible to COVID-19" (Q1), while only $41.4 \%$ of patients taking 5-ASA agreed with Q1 ( $<<0.001$ ). The agreement for the perceived risk of severe lung injury (Q2) was significantly pronounced in patients receiving biologic monotherapy or JAK inhibitors $(42.6 \%, \mathrm{p}=0.005)$. The proportion of patients who agreed that "during the COVID-19 pandemic, IBD patients should avoid hospital visits, even if their disease worsened" (Q4) was significantly higher in patients taking biologic monotherapy or JAK inhibitors (31.1\%) and those on immunomodulator monotherapy (27.5\%) compared to those taking 5-ASA alone $(10.8 \%)(\mathrm{p}<0.001)$. Among patients on immunomodulator monotherapy, $14.5 \%$ agreed that "to prevent COVID-19 infection, IBD patients should withhold IBD drugs" (Q5), whereas the agreement with this statement
(Q5) among patients taking other drugs was only $2.6 \%$ to $5.7 \%(\mathrm{p}<0.001)$. Similarly, the biologics combined with immunomodulator group mostly agreed with the statement that "if patients are infected by COVID-19, IBD drugs should be discontinued" (Q6) (27.9\%, p $<0.001)$. However, the degree of agreement to concerns about intestinal damage (Q3) and the protective effects of pneumococcal vaccination (Q7) were not statistically different according to the medication type (Fig. 3).

When the degree of agreement regarding perceptions was analyzed according to each patients' disease activity, the perceived risk for COVID-19 on intestinal damage in IBD patients (Q3) was significantly higher in patients with moderate to severe disease activity than in those with remission to mild disease activity ( $31.9 \%$ vs $31.2 \%, \mathrm{p}=0.003$ ). 
Q1. IBD patients are more susceptible to COVID-19

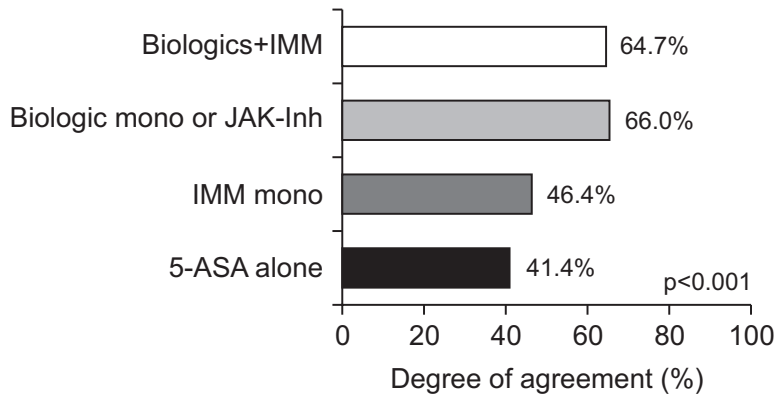

Q3. COVID-19 can cause intestinal damage in IBD patients

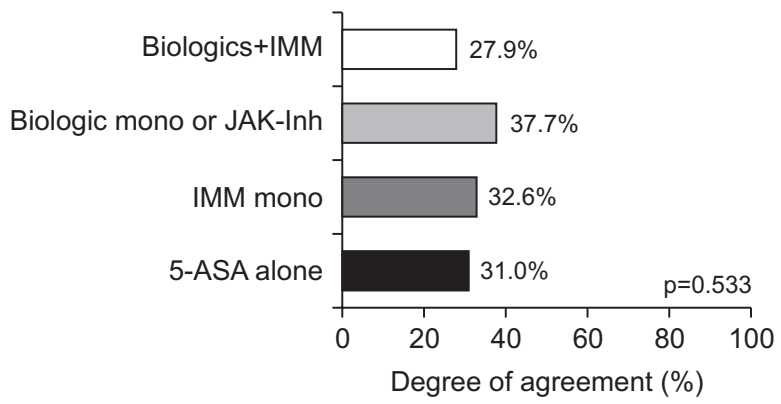

Q5. To prevent COVID-19, IBD patients should withhold IBD drugs

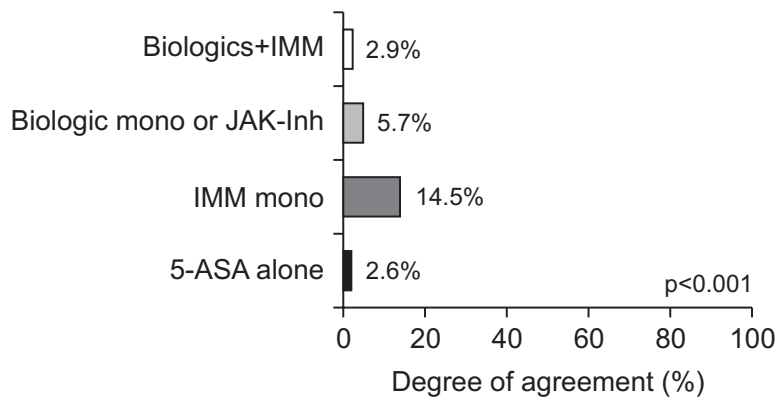

Q7. Pneumococcal vaccination will help from progression to severe pneumonia when infected with SARS-CoV-2

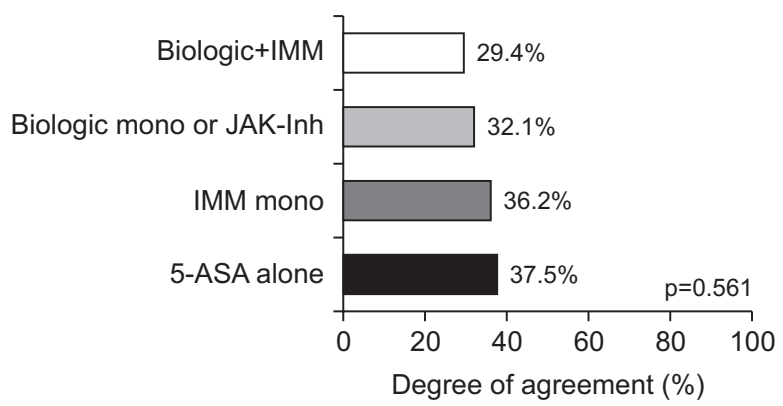

Q2. COVID-19 can cause more severe lung injury in IBD patients

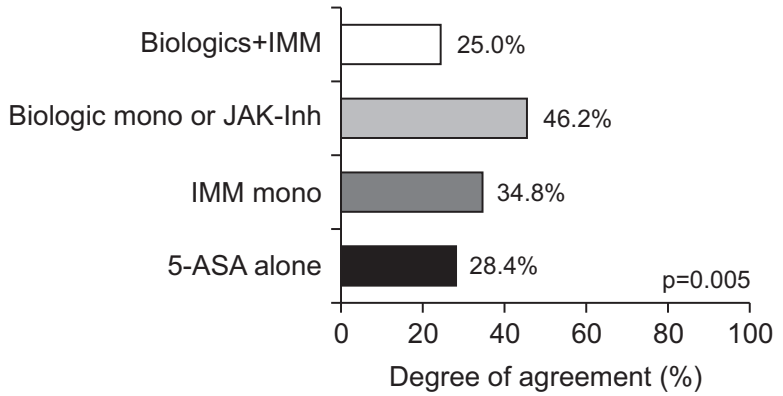

Q4. During the COVID-19 pandemic, IBD patients should avoid hospital visits, even if their disease worsened

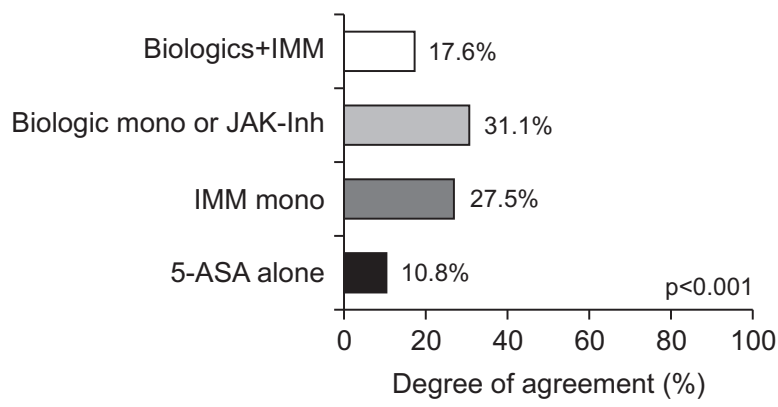

Q6. If patients are infected with SARS-CoV-2, IBD drugs should be discontinued

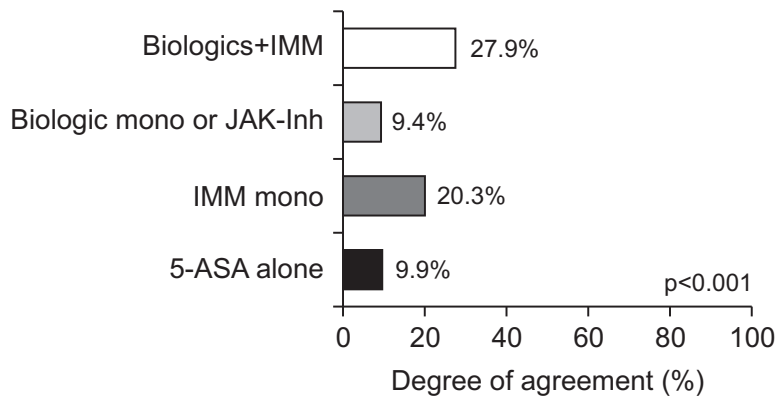

Fig. 3. Proportions of responders showing agree or strongly agree to the patient's perception questionnaire (Q1-7) according to the medication type. IBD, inflammatory bowel disease; COVID-19, coronavirus disease 2019; IMM, immunomodulator; mono, monotherapy; JAK-inh, Janus kinaseinhibitor; 5-ASA, 5-aminosalicylate; SARS-CoV-2, severe acute respiratory syndrome coronavirus 2.

More patients with remission to mild disease activity agreed that the "pneumococcal vaccination will help minimize the risk of progression to severe pneumonia when infected with COVID-19" (Q7) compared with patients with moderate to severe disease activity $(35.9 \%$ vs $14.3 \%$, $\mathrm{p}=0.041)$. However, the degree of agreement with other questions (Q1, Q2, Q4, and Q5), including prophylactic discontinuation of IBD medications to prevent COVID-19, 
was not statistically different according to the patients' disease activity (data not shown).

\section{DISCUSSION}

In this study, we found that COVID-19 had a great influence on the daily life and medical utilization of IBD patients in South Korea. Although patients with IBD were considered to be susceptible to COVID-19, most felt that they should continue their IBD drugs. However, the patients taking biologics/JAK inhibitors or biologics combined with immunomodulator expressed more concerns about their susceptibility and the risks of their drugs in relation to the COVID-19 infection than those not taking these drugs. Despite these concerns about the risk of infection due to their underlying disease or medications, few discussed their concerns with the medical staff.

Half of the IBD patients (50.4\%) felt that they were more susceptible to COVID-19. Recent surveys also showed that these respondents considered the risk of acquiring severe COVID-19 and were fearful of this happening. ${ }^{4-6}$ These results are not surprising because IBD patients have a higher risk of acquiring influenza compared with the non-IBD cohort. ${ }^{7}$ However, to date, there is no evidence of increased risk of COVID-19 infection in IBD patients than that in the general population. ${ }^{8,9}$ In a large population-based cohort in the United States, the risk of severe COVID-19 in patients with IBD was similar to that in patients without IBD. $^{10}$

IBD patients in our survey strictly adhered to personal protection guidance during the COVID-19 crisis. This is in line with the recommendation by the European Crohn's and Colitis Organisation, which states that patients with IBD should follow the national recommendations; in addition, they required additional caution. ${ }^{11}$ None of the IBD patients in our cohort who contacted the investigators were infected with COVID-19.

In our study, only $43.5 \%$ of patients who experienced suspected symptoms of COVID-19 had discussed those symptoms with a physician. The low rate of consultations with physicians may be explained by the social liability of patients during the early COVID-19 outbreak. The early outbreak of COVID-19 in South Korea was closely linked to certain religious groups called Shincheonji, who received substantial social criticism for not cooperating with the epidemiological investigations. ${ }^{12}$ Thus, reporting symptoms of COVID-19 at that time could have been distressing for the patients by drawing negative attention related to Shincheonji. Although most of these negative perceptions of patients confirmed with COVID-19 have now disappeared, medical staff should encourage patients to report and get tested quickly for suspected symptoms of COVID-19. A recent meta-analysis revealed that the clinical presentations of COVID-19 in patients with IBD were similar to those of the general population, but diarrhea occurred in a quarter of IBD patients. ${ }^{13}$ Because diarrhea is common during IBD exacerbations, it can be easily overlooked. Therefore, medical staff should strive to actively communicate with patients so that patients can immediately report even minor symptoms such as diarrhea.

In line with other studies, IBD patients believed that they needed to adhere to their medications during the COVID-19 pandemic. ${ }^{5,14}$ However, there was a disparity between patients' perceptions and coping aspects of drug adherence. Over $80 \%$ of patients disagreed that they should postpone or withhold IBD drugs to prevent COVID-19, yet a substantial proportion of them was nonadherent. We also found that among patients who demonstrated a disparity between their perceptions and coping aspects, a notable number of patients using biologics or JAK inhibitors (39.4\%) did not adhere to their drug due to fears of COVID-19 infection (Fig. 3). These findings could be explained in part by the results of the subgroup analysis of patients' perceptions according to drug type. Notably, there were significant differences in patients' perceptions of the risk of COVID-19 infection from IBD itself and medications according to specific drug classes. The negative perceptions about the effects of IBD and drugs on COVID-19 infection were significantly higher among patients treated with immunosuppressive therapy than among those treated with 5-ASA alone. Negative perceptions among patients using immunosuppressive therapies may be natural, because studies have repeatedly shown that IBD patients using a biologic and/or immunosuppressant have an increased risk of serious infection. ${ }^{15-17}$ However, additional evidence is needed to determine whether the general vulnerability of patients with immunosuppressive therapy to infection applies equally to COVID-19. Additionally, when patients' perceptions were analyzed according to the IBD disease activity, their perceptions regarding the necessity of prophylactic drug discontinuation did not differ according to disease activity. As active IBD itself is known to be a risk factor for severe COVID-19, medical staff should encourage IBD patients to maintain their medications even during the COVID-19 epidemic. ${ }^{18}$

To date, empirical guidance on IBD management has been issued by several societies during the COVID-19 pandemic. Although there are still limited data on the safety of IBD drugs, all experts and societies recommend continuing IBD-specific treatment since it has been recognized that the risk of active disease is higher than the uncertain 
risk of immunosuppression, which tends to increase the risk of COVID-19. ${ }^{8,19-21}$ Although early data have reported that combination therapy and thiopurine monotherapy may be associated with a higher risk of severe COVID-19 infection in IBD patients, ${ }^{22}$ supporting evidence regarding the discontinuation of thiopurine in patients on stable maintenance is still very weak..$^{19,20}$

Considering that only $7 \%$ of patients discussed the safety of their drugs with medical staff, we also need to pay attention to the effect of the lack of communication between patients and the medical staff. Neglecting treatment due to misinformation can exacerbate IBD. Thus, medical staff should strengthen communications with patients and provide appropriate health-related guidance to prevent abrupt termination of IBD-specific treatment during epidemics. Additionally, a lower level of knowledge about COVID-19 is reported to be associated with anxiety in patients with IBD. ${ }^{23}$ Therefore, physicians should take a more delicate approach from the perspective of IBD patients to reduce their anxiety and improve their attitude toward COVID-19 and its prevention.

Our results also highlight the need for reinforced education for vaccination strategies in patients with IBD. The answers for the positive effect of pneumococcal vaccination during COVID-19 (Q7) varied widely. The World Health Organization announced that pneumococcal vaccination does not prevent COVID-19 infection. ${ }^{24}$ However, the European Crohn's and Colitis Organisation-COVID Taskforce recently recommended pneumococcal vaccination to avoid additional pulmonary comorbidities during the COVID-19 pandemic. ${ }^{11}$ Thus, medical staff should provide accurate information on the benefits of pneumococcal vaccination in our patients.

To enhance active communications, physicians can use social media and other technologies which have already been widely used by patients with IBD. ${ }^{25}$ According to a recent survey of IBD patients in the United Kingdom, young people preferred application or video consultations, while those over 55 preferred phone consultation or faceto-face consultations. ${ }^{26}$ Therefore, for patients who missed appointments for a considerable period of time, especially the elderly, telephone notifications from physicians or specialist nurses would be of great benefit in communicating with the patients. Additionally, one of the best ways to educate patients in the era of COVID-19 is to make videos for sharing platforms such as YouTube by academy or expert groups, and to have medical staff actively promote them to patients.

Recently, telemedicine has been suggested as a safety strategy to prevent disease transmission in the era of CO-
VID- $19 .{ }^{27}$ Interestingly, only $13 \%$ of patients in this study reported using telemedicine. Furthermore, the majority (71.6\%) of patients who had experienced telemedicine answered that they had to subsequently visit a pharmacy near the hospital. This result indicated that the temporarily allowed telemedicine did not reduce the frequency of patients leaving their homes. Thus, the implementation of telemedicine should be individualized depending on the patient's medical condition and the resource availability of each institution.

Our study has several limitations. First, this was not a controlled study. Second, objective IBD activities at the time of the survey were not available. However, to compensate for this, only patients with records of disease activity within 3 months of evaluation were enrolled in this study.

The strength of our study is that a relatively large number of IBD patients completed the survey in the major epidemic area during the early COVID-19 crisis in South Korea. Since it was a COVID-19 epidemic area where patient access to medical care was limited for various reasons, the method of combining online and telephone surveys rather than targeting outpatients was used to reduce selection bias. As a result, the current survey achieved a relatively high response rate (61.9\%) compared to previous studies. ${ }^{4,5,26}$

In summary, most patients with IBD adhered strictly to the general principles for the prevention of epidemics. Although there were concerns about the increased risk of COVID-19 among IBD patients, most patients believed that maintaining their treatment is important. However, a substantial number of IBD patients withheld their medication without consulting medical staff due to concerns related to COVID-19. This discrepancy highlights the need for medical personnel to communicate appropriate information regarding the disease or medication and the risk of COVID-19 with their patients.

\section{CONFLICTS OF INTEREST}

No potential conflict of interest relevant to this article was reported.

\section{ACKNOWLEDGEMENTS}

This study was supported by a research grant from the Daegu Medical Association COVID-19 Scientific Committee. 


\section{AUTHOR CONTRIBUTIONS}

Conceptualization: K.O.K. Data curation: Y.J.L. Formal analysis: Y.J.L. Funding acquisition: K.O.K. Methodology: K.O.K., Y.J.L. Project administration: Y.J.L., M.C.K. Visualization: K.B.C., K.S.P., B.I.J. Writing - original draft: Y.J.L. Writing - review \& editing: K.O.K., M.C.K., K.B.C., K.S.P., B.I.J. Approval of final manuscript: all authors.

\section{ORCID}

Yoo Jin Lee Kyeong Ok Kim Min Cheol Kim Kwang Bum Cho Kyung Sik Park Byeong Ik Jang https://orcid.org/0000-0003-1799-0146 https://orcid.org/0000-0001-5799-7436 https://orcid.org/0000-0002-2234-8070 https://orcid.org/0000-0003-2203-102X https://orcid.org/0000-0003-1874-9936 https://orcid.org/0000-0002-3037-9272

\section{SUPPLEMENTARY MATERIALS}

Supplementary materials can be accessed at https://doi. org/10.5009/gnl210064.

\section{REFERENCES}

1. Lee JY, Hong SW, Hyun M, et al. Epidemiological and clinical characteristics of coronavirus disease 2019 in Daegu, South Korea. Int J Infect Dis 2020;98:462-466.

2. Chung HS, Lee DE, Kim JK, et al. Revised triage and surveillance protocols for temporary emergency department closures in tertiary hospitals as a response to COVID-19 crisis in Daegu metropolitan city. J Korean Med Sci 2020;35:e189.

3. Satsangi J, Silverberg MS, Vermeire S, Colombel JF. The Montreal classification of inflammatory bowel disease: controversies, consensus, and implications. Gut 2006;55:749753.

4. Goodsall TM, Han S, Bryant RV. Understanding attitudes, concerns, and health behaviors of patients with inflammatory bowel disease during the coronavirus disease 2019 pandemic. J Gastroenterol Hepatol 2021;36:1550-1555.

5. Grunert PC, Reuken PA, Stallhofer J, Teich N, Stallmach A. Inflammatory bowel disease in the COVID-19 pandemic: the patients' perspective. J Crohns Colitis 2020;14:17021708.

6. D’Amico F, Rahier JF, Leone S, Peyrin-Biroulet L, Danese S. Views of patients with inflammatory bowel disease on the COVID-19 pandemic: a global survey. Lancet Gastroenterol Hepatol 2020;5:631-632.
7. Tinsley A, Navabi S, Williams ED, et al. Increased risk of influenza and influenza-related complications among 140,480 patients with inflammatory bowel disease. Inflamm Bowel Dis 2019;25:369-376.

8. Rubin DT, Feuerstein JD, Wang AY, Cohen RD. AGA clinical practice update on management of inflammatory bowel disease during the COVID-19 pandemic: expert commentary. Gastroenterology 2020;159:350-357.

9. Taxonera C, Sagastagoitia I, Alba C, Mañas N, Olivares D, Rey E. 2019 novel coronavirus disease (COVID-19) in patients with inflammatory bowel diseases. Aliment Pharmacol Ther 2020;52:276-283.

10. Singh S, Khan A, Chowdhry M, Bilal M, Kochhar GS, Clarke K. Risk of severe coronavirus disease 2019 in patients with inflammatory bowel disease in the United States: a multicenter research network study. Gastroenterology 2020;159:1575-1578.

11. Magro F, Rahier JF, Abreu C, et al. Inflammatory bowel disease management during the COVID-19 outbreak: the ten do's and don'ts from the ECCO-COVID Taskforce. J Crohns Colitis 2020;14(Supplement_3):S798-S806.

12. Kim HJ, Hwang HS, Choi YH, et al. The delay in confirming COVID-19 cases linked to a religious group in Korea. J Prev Med Public Health 2020;53:164-167.

13. Singh AK, Jena A, Kumar-M P, Jha DK, Sharma V. Clinical presentation of COVID-19 in patients with inflammatory bowel disease: a systematic review and meta-analysis. Intest Res. Epub 2021 Jan 18. https://doi.org/10.5217/ir.2020.00108.

14. Trindade IA, Ferreira NB. COVID-19 pandemic's effects on disease and psychological outcomes of people with inflammatory bowel disease in Portugal: a preliminary research. Inflamm Bowel Dis 2021;27:1224-1229.

15. Singh S, Facciorusso A, Dulai PS, Jairath V, Sandborn WJ. Comparative risk of serious infections with biologic and/or immunosuppressive therapy in patients with inflammatory bowel diseases: a systematic review and meta-analysis. Clin Gastroenterol Hepatol 2020;18:69-81.

16. Khan N, Patel D, Trivedi C, et al. Overall and comparative risk of herpes zoster with pharmacotherapy for inflammatory bowel diseases: a nationwide cohort study. Clin Gastroenterol Hepatol 2018;16:1919-1927.

17. Wisniewski A, Kirchgesner J, Seksik P, et al. Increased incidence of systemic serious viral infections in patients with inflammatory bowel disease associates with active disease and use of thiopurines. United European Gastroenterol J 2019;8:303-313.

18. Bezzio C, Saibeni S, Variola A, et al. Outcomes of COVID-19 in 79 patients with IBD in Italy: an IG-IBD study. Gut 2020;69:1213-1217.

19. Kennedy NA, Jones GR, Lamb CA, et al. British Society of Gastroenterology guidance for management of inflamma- 
tory bowel disease during the COVID-19 pandemic. Gut 2020;69:984-990.

20. Kim KO, Jang BI. Management of inflammatory bowel disease in the COVID-19 era. Intest Res. Epub 2021 Feb 3. https://doi.org/10.5217/ir.2020.00156.

21. D’Amico F, Danese S, Peyrin-Biroulet L; ECCO COVID Taskforce. Inflammatory bowel disease management during the coronavirus-19 outbreak: a survey from the European Crohn's and Colitis Organization. Gastroenterology 2020;159:14-19.

22. Ungaro RC, Brenner EJ, Gearry RB, et al. Effect of IBD medications on COVID-19 outcomes: results from an international registry. Gut 2021;70:725-732.

23. Spagnuolo R, Larussa T, Iannelli C, et al. COVID-19 and inflammatory bowel disease: patient knowledge and perceptions in a single center survey. Medicina (Kaunas) 2020;56:407.
24. World Health Organization (WHO). Advice for the public mythbusters, FACT: Vaccines against pneumonia DO NOT protect against the COVID-19 virus [Internet]. Geneva: WHO; c2020 [cited 2021 May 25]. https://www.who.int/ emergencies/diseases/novel-coronavirus-2019/advice-forpublic/myth-busters.

25. Ahmed W, Taft TH, Charabaty A. Social media in inflammatory bowel disease: the patient and physician perspective. Curr Opin Gastroenterol 2021;37:328-335.

26. Harris RJ, Downey L, Smith TR, Cummings JR, Felwick R, Gwiggner M. Life in lockdown: experiences of patients with IBD during COVID-19. BMJ Open Gastroenterol 2020;7:e000541.

27. Danese S, Sands B, Ng SC, Peyrin-Biroulet L. The day after COVID-19 in IBD: how to go back to 'normal'. Nat Rev Gastroenterol Hepatol 2020;17:441-443. 